\title{
On the pre-main sequence circularization period ${ }^{\star}$
}

\author{
C. H. F. Melo ${ }^{1}$, E. Covino ${ }^{2}$, J. M. Alcalá ${ }^{2}$, and G. Torres ${ }^{3}$ \\ 1 Observatoire de Genève, Ch. des Maillettes 51, 1290 Sauverny, Switzerland \\ 2 Osservatorio Astronomico di Capodimonte, Via Moiariello 16, 80131 Napoli, Italy \\ 3 Harvard-Smithsonian Center for Astrophysics, 60 Garden Street, Cambridge, MA 02138, USA
}

Received 9 February 2001 / Accepted 3 September 2001

\begin{abstract}
In this paper we present an updated compilation of the currently known pre-main sequence (PMS) spectroscopic binaries (SB) for which orbital elements have been determined. In particular we report our results for and discuss the case of the bona-fide PMS system RX J1603.9-3938, which has a circular orbit and a period of 7.56 days. This is the longest orbital period for a circular orbit found among the bona-fide PMS spectroscopic binaries so far, and we suggest that this system may be thus considered to now set the PMS circularization period. The longer period is compatible with the circularization periods already known for older binary populations such as the Hyades and Praesepe. When considered in the context of the circularization periods for other binary populations of different ages, the new PMS circularization period strongly supports the suggestion of Mathieu et al. (1992) that a hybrid scenario (tidal circularization occurring on both PMS phase and on MS phase) could explain the observed circularization periods as a function of age. The apparently circular orbit observed in another PMS system, RX J1301.0-7654a, with an orbital period of nearly 13 days, may perhaps change this picture when the orbit is improved and the system is better understood.
\end{abstract}

Key words. stars: binaries: spectroscopic - stars: pre-main sequence - stars: individual: RX J1603.9-3938

\section{Introduction}

The distribution of orbital eccentricities versus orbital period has now been studied for binaries of several coeval samples. In all cases a common feature is observed, i.e., binaries with orbital period shorter than a critical period, called the circularization period $\left(P_{\text {circ }}\right)$, have circular orbits, while the wide binaries have significant orbital eccentricities. This common feature of an $e-\log P$ diagram is usually interpreted as evidence that tidal interactions are driving the orbital evolution. Two distinct mechanisms are usually invoked to explain orbital circularization. In the first one, the tidal-torque mechanism, viscosity will make tides lag (or precede) the line joining the centers of the two components, and this misalignment will generate a torque which will be responsible for the circularization of the orbit (Zahn 1966; Zahn 1977). A key issue in describing tidal-torque interaction is the treatment of the viscosity which has been subject to much discussion. It dictates how strong the dissipative mechanisms are. In terms of orbital period, for a given amount of time, the stronger the dissipative mechanisms are, the longer will be the longest

\footnotetext{
Send offprint requests to: C. H. F. Melo,

e-mail: Claudio.Melo@obs.unige.ch

* Based on observations carried out at the Swiss Euler Telescope at ESO, La Silla, Chile.
}

orbital period presenting circular orbit (i.e., the circularization period as defined by Duquennoy et al. 1992). Zahn (1977) (see also Zahn 1989; Goldman \& Mazeh 1991) identifies the turbulent friction and the radiative damping as being the main source of viscosity in late- and early-type stars, respectively.

The second mechanism, hydrodynamical mechanism, was suggested by Tassoul in a series of papers (see Tassoul 1995; Tassoul 2000 and references therein). It involves large-scale hydrodynamical flows within the nonsynchronous tidally distorted component. Their existence is always required to satisfy the stress-free condition in the star surface. These flows will replace high angular velocity fluid by low angular velocity fluid, braking the star.

Regardless of the mechanism believed to act, the time required to circularize orbits, $t_{\text {circ }}$, up to a critical period $P_{\text {circ }}$ will have the same functional relation $t_{\text {circ }} \propto P_{\text {circ }}^{\gamma}$. The choice of the mechanism (and dissipation prescription for the tidal-torque mechanism) is reflected by the value of $\gamma$. The relative sensitivity of $P_{\text {circ }}$ to stellar age motivated Mathieu \& Mazeh (1988) to propose that the observed circularization period for a coeval sample of binaries could in fact be used as a clock to determine the age of galactic clusters. A different approach was proposed by Zahn \& Bouchet (1989). These authors, following the suggestion of Mayor \& Mermilliod (1984) that the circularization of 
the orbit would occur very fast during the PMS phase, integrated the full set of equations governing the tidal evolution for binary systems with masses ranging from 0.5 to $1.25 M_{\odot}$ from the birth-line up to $10^{10}$ years. They found that most of the orbital circularization occurs during the pre-main sequence phase, primarily near the stellar birthline. According to their results, the circularization period set during the PMS phase is about 7-8.5 days, depending on stellar mass. In addition, as no further significant circularization occurs on the main-sequence, they concluded that the circularization period for all binary populations (age $<10^{10}$ years) should be equal to that established during the PMS phase. Therefore, a determination of the circularization period for binary populations of different ages is needed to enable us to test both the different dissipation prescriptions and the effectiveness of the PMS tidal circularization.

While the circularization period is determined for some young cluster binary populations and for the two older binary populations of M 67 and of the Galactic Halo, for the PMS binaries Mathieu (1994) counted only 25 spectroscopic binaries with known orbital elements. These numbers have been increasing in the last years thanks to many WTTS found by the ROSAT X-ray all-sky survey in the nearby star-forming regions. The preliminary results of on-going spectroscopic monitoring surveys on these stars (Covino et al. 2001a) reveal that many of them are in fact spectroscopic binaries.

In this paper we present a new PMS binary system with orbital parameters that make it a crucial system for testing theory. We confront both Mathieu \& Mazeh's and Zahn \& Bouchet's hypothesis to the new available data on PMS spectroscopic binaries to review the problem of tidal interactions.

\section{The observational data}

\subsection{The system RX J1603.9-3938}

In Table 1 we present radial velocity observations for the double-lined spectroscopic binary RX J1603.9-3938¹ carried out with the spectrograph CORALIE attached to the Swiss Euler Telescope at ESO, La Silla. The radial velocity curve is shown in Fig. 1 and the respective orbital elements and stellar parameters for both components are given in Table 2. The system has a circular orbit and an orbital period of 7.56 days. Its barycentric velocity $\left(\gamma=1.921 \mathrm{~km} \mathrm{~s}^{-1}\right)$ is in agreement with the mean radial velocity $\left(<V_{\text {rad }}>=-0.03 \pm 1.2 \mathrm{~km} \mathrm{~s}^{-1}\right)$ of the 9 Lupus bona-fide CTTS and with the mean radial velocity $\left(<V_{\text {rad }}>=1.29 \pm 0.87 \mathrm{~km} \mathrm{~s}^{-1}\right)$ of the "on-cloud" Lupus ROSAT WTTS both listed in Wichmann et al. (1999).

Determination of luminosities and spectral types were obtained for both components through a matching of the binary spectrum with that of a synthetic binary following

1 During the writing of this paper we learned that Guenther et al. (2001) independently derived an orbital solution for this system.

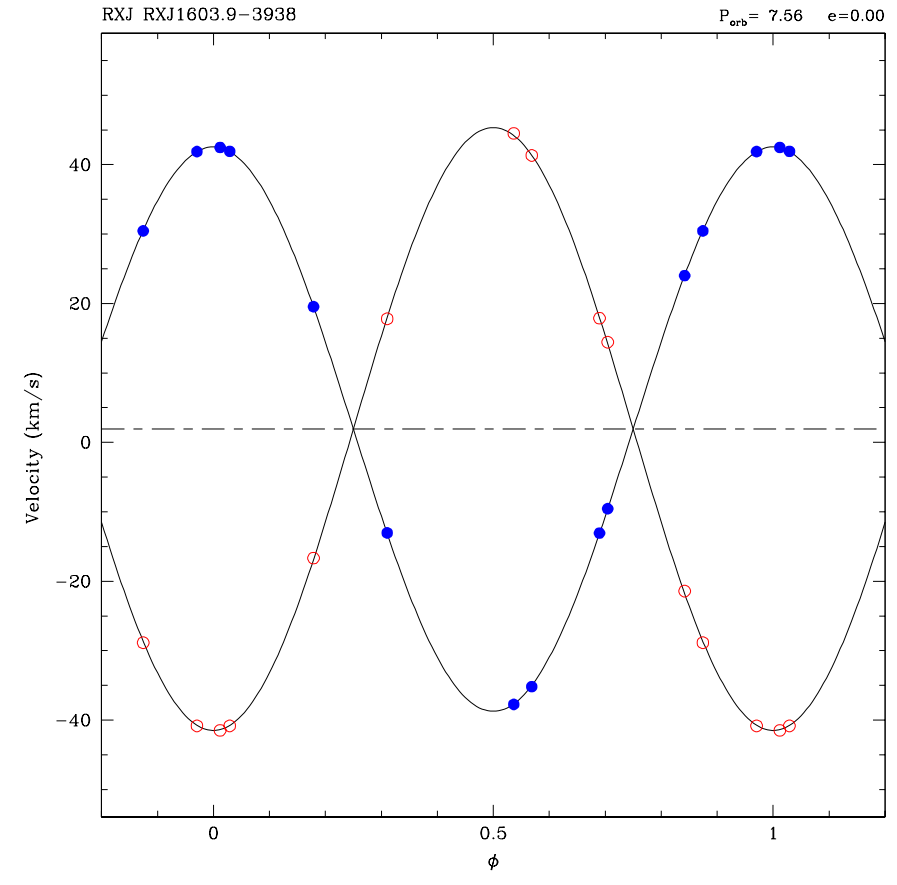

Fig. 1. Radial velocity curve of RX J1603.9-3938. Radial velocities observations for the primary and secondary components are drawn as a filled and open circles, respectively.

the prescription of Lee et al. (1994). Details on the application of the matching technique and on the radial and rotational velocities determination are given in Covino et al. (2000, 2001a). Once individual luminosities have been derived, we can estimate the true Li I $\lambda 6708 \AA$ equivalent width for each component (i.e. the equivalent width corrected from the veiling due to continuum of the companion). The values given in Table 2 show that the Li I content in both components of RX J1603.9-3938 are higher than those observed in stars of same spectral type at the age of Pleiades ( $\sim 350 \mathrm{~m} \AA$ for $\mathrm{K} 2$ and $\sim 320 \mathrm{~m} \AA$ for a K4). Finally, ages, masses and the radii for both components were determined from evolutionary models by D'Antona \& Mazzitelli (1997). The choice of using the evolutionary models by D'Antona \& Mazzitelli to derive ages and radii is completely arbitrary since there is no standard PMS evolutionary model. The reader should be aware that other models can yield different masses and ages (e.g., Baraffe et al. 1998; see e.g., Simon et al. 2000; Covino et al. 2001a for a comparison between different evolutionary tracks). However, we stress that the use of other sets of PMS tracks does not change the main conclusions of this paper. Together, the Li content of each component, the barycentric velocity of the system and its age strongly suggest that both components of RX J1603.9-3938 are in fact bona-fide T-Tauri stars.

\subsection{Data available for other PMS systems}

The orbital period and eccentricity for the PMS binary systems known to the authors are listed in Table 3 . When available in respective reference, the spectral type of the 
Table 1. Radial Velocity data for RX J1603.9-3938. Column 1: heliocentric julian date; Col. 2: orbital phase; Col. 3: radial velocity measurements for the primary star; Col. 4: error on the radial velocity (primary); Col. 5: residuals (primary); Col. 6: radial velocity measurements for the secondary star; Col. 7: error on the radial velocity (secondary); Col. 8: residuals (secondary). Columns 3 to 8 are given in $\mathrm{km} \mathrm{s}^{-1}$.

\begin{tabular}{ccrrrrrr}
\hline HJD & Phase & \multicolumn{1}{c}{$V r_{\mathrm{a}}$} & \multicolumn{1}{c}{$\sigma$} & \multicolumn{1}{c}{$\mathrm{O}-\mathrm{C}$} & \multicolumn{1}{c}{$V r_{\mathrm{b}}$} & \multicolumn{1}{c}{$\sigma$} & \multicolumn{1}{c}{$\mathrm{O}-\mathrm{C}$} \\
\hline 51209.8218 & 0.1788 & 19.54 & 0.02 & 0.03 & -16.67 & 0.05 & 0.20 \\
51210.8160 & 0.3103 & -13.04 & 0.04 & 0.08 & 17.80 & 0.08 & -0.20 \\
51212.7694 & 0.5688 & -35.19 & 0.04 & -0.21 & 41.33 & 0.07 & -0.01 \\
51213.7936 & 0.7044 & -9.56 & 0.02 & 0.02 & 14.43 & 0.05 & 0.23 \\
51214.8325 & 0.8418 & 24.02 & 0.03 & -0.07 & -21.42 & 0.06 & 0.34 \\
51215.8039 & 0.9704 & 41.87 & 0.04 & 0.01 & -40.83 & 0.08 & -0.01 \\
51253.9018 & 0.0119 & 42.48 & 0.75 & 0.03 & -41.48 & 0.75 & -0.11 \\
51257.8665 & 0.5366 & -37.73 & 0.75 & -0.08 & 44.50 & 0.75 & 0.31 \\
51311.9213 & 0.6897 & -13.06 & 0.04 & 0.05 & 17.87 & 0.08 & -0.10 \\
51320.8742 & 0.8745 & 30.45 & 0.03 & -0.11 & -28.85 & 0.05 & -0.18 \\
51684.7691 & 0.0291 & 41.91 & 0.02 & 0.03 & -40.83 & 0.03 & -0.07 \\
\hline
\end{tabular}

Table 2. Orbital and stellar parameters for RX J1603.9-3938.

\begin{tabular}{|c|c|c|}
\hline & $e \equiv 0$ & $e$ free \\
\hline$P_{\text {orb }}($ days $)$ & $7.56679 \pm 0.00015$ & $7.55678 \pm 0.00019$ \\
\hline$T_{0}-2400000^{1}$ (HJD) & $51004.4375 \pm 0.0049$ & $51003.5 \pm 1.8$ \\
\hline$e$ & 0 . & $0.0009 \pm 0.0014$ \\
\hline$\gamma\left(\mathrm{km} \mathrm{s}^{-1}\right)$ & $1.921 \pm 0.034$ & $1.925 \pm 0.036$ \\
\hline$K_{\mathrm{a}}\left(\mathrm{km} \mathrm{s}^{-1}\right)$ & $40.643 \pm 0.060$ & $40.624 \pm 0.068$ \\
\hline$K_{\mathrm{b}}\left(\mathrm{km} \mathrm{s}^{-1}\right)$ & $43.411 \pm 0.080$ & $43.395 \pm 0.086$ \\
\hline$a_{\mathrm{a}} \sin i\left(10^{9} \mathrm{~m}\right)$ & $4.2234 \pm 0.0063$ & $4.2214 \pm 0.0071$ \\
\hline$a_{\mathrm{b}} \sin i\left(10^{9} \mathrm{~m}\right)$ & $4.5110 \pm 0.0082$ & $4.5093 \pm 0.0090$ \\
\hline$M_{\mathrm{a}} \sin ^{3} i\left(M_{\odot}\right)$ & $0.24069 \pm 0.00091$ & $0.24041 \pm 0.00100$ \\
\hline$M_{\mathrm{b}} \sin ^{3} i\left(M_{\odot}\right)$ & $0.22535 \pm 0.00082$ & $0.22506 \pm 0.00091$ \\
\hline$q=M_{\mathrm{b}} / M_{\mathrm{a}}$ & $0.936 \pm 0.003$ & $0.936 \pm 0.004$ \\
\hline$\sigma(\mathrm{O}-\mathrm{C})\left(\mathrm{km} \mathrm{s}^{-1}\right)$ & 0.143 & 0.145 \\
\hline Number of measur. & 11 & 11 \\
\hline & Primary (a) & Secondary (b) \\
\hline$V \sin i\left(\mathrm{~km} \mathrm{~s}^{-1}\right)$ & 2. & 5 \\
\hline$L\left(L_{\odot}\right)$ & 0.72 & 0.53 \\
\hline $\log T_{\text {eff }}(\mathrm{K})$ & 3.678 & 3.664 \\
\hline$S T$ & $\mathrm{~K} 2$ & K4 \\
\hline$E W_{\operatorname{Li} \lambda 6708}(\mathrm{~m} \AA)$ & 420 & 390 \\
\hline$R\left(R_{\odot}\right)$ & 1.25 & 1.19 \\
\hline$M\left(M_{\odot}\right)$ & 1.1 & 0.9 \\
\hline age (Myr) & 7 & 7 \\
\hline
\end{tabular}

${ }^{1}$ For the eccentric case, $T_{0}$ gives the time of periastron passage while for the circular case, $T_{0}$ indicates the time of the radial velocity maximum of the primary star.

components is given. When only the effective temperature is available we use the calibration of de Jager \& Nieuwenhuijzen (1987) to transform effective temperatures into spectral types. We also mark the systems in which at least one of the components is not within the mass range investigated in Zahn \& Bouchet (1989). The nature of the spectroscopic binary system (i.e., single- or double-lined) is indicated as well. Comparing our Table 3 to Mathieu's (1994) Tables A1 and A2, we list 15 new entries. Also, some entries listed in Mathieu (1994) had their reference updated, but a few of them still remain unpublished. In Table 4 we list age and circularization period with their respective references for other low-mass binary populations for which the circularization period is determined.

\section{Results and discussion}

\subsection{The updated PMS e - $\log P$ diagram and the new PMS circularization period}

An updated version of the PMS $e-\log P$ diagram is shown in Fig. 2. Circles stand for double-lined spectroscopic binary (SB2) systems and triangles stand for single-lined spectroscopic binary (SB1) systems. Those SB systems where at least one of the components has a mass clearly outside the range explored by Zahn \& Bouchet (1989), i.e. from 0.5 to $1.5 M_{\odot}$, are shown as open symbols.

The main difference between our Fig. 2 and Fig. 2a of Mathieu (1994) is the addition of 13 new SB systems with $P_{\text {orb }}<100$ days and 2 systems with $P_{\text {orb }}>100$ days. The fact that the majority of the newly found systems are short-period, equal mass, and low to moderate eccentricity binaries can be understood as an observational bias since these systems are easier to be detected. Nevertheless, we can use these new short-period SB systems to revisit the important issue of the PMS circularization period.

The circularization period for a binary population is defined as the longest period for a circular orbit (Duquennoy et al. 1992). An inspection of Fig. 2 and/or of Table 3 indicates, therefore, that the PMS circularization period would now appear to be set by RX J1301.0-7654a $\left(P_{\text {orb }}=13.1\right.$ days $)$, assuming that its orbit is truly circular. The theory of the tidal interaction predicts that the synchronization between rotational and orbital motion is typically achieved more rapidly than orbital circularization $\left(t_{\text {circ }} \sim 10 \times t_{\text {sync }} \sim 10^{6}\right.$ years) (e.g.: Zahn 1977 ; 
Table 3. Orbital period and eccentricity for the known PMS spectroscopic binaries. When possible, information about the spectral type of the components is given. Spatial location and the respective reference are also indicated.

\begin{tabular}{|c|c|c|c|c|c|}
\hline Star & $\begin{array}{l}\text { Period } \\
\text { (days) }\end{array}$ & $e$ & $\begin{array}{c}\text { Spectral Type } \\
\text { (Primary+Secondary) }\end{array}$ & Location & Reference \\
\hline HD $155555^{2}$ & 1.68 & 0 & $\mathrm{G} 5+\mathrm{K} 1$ & Isolated & Pasquini et al. (1991) \\
\hline $\mathrm{RS} \mathrm{Cha}^{2}$ & 1.67 & 0 & ${ }^{\dagger} \mathrm{A} 8+\mathrm{A} 8$ & Cha & Andersen (1975) \\
\hline V4046Sgr ${ }^{2}$ & 2.42 & 0 & $\mathrm{~K} 5+\mathrm{K} 7$ & Isolated & de la Reza et al. (1986) \\
\hline $155913-2233^{1}$ & 2.42 & 0 & K5 & Sco-Cen & Mathieu et al. (1989) \\
\hline $\mathrm{TY} \mathrm{CrA}^{2,3}$ & 2.89 & 0 & ${ }^{\dagger} \mathrm{B} 9$ & Corona Aus & Casey et al. (1993) \\
\hline RX J0529.4+0041 ${ }^{2}$ & 3.03 & 0 & $\mathrm{~K} 1+\mathrm{K} 7$ & Orion & Covino et al. (2000) \\
\hline $\mathrm{V} 826 \mathrm{Tau}^{2}$ & 3.88 & 0 & $\mathrm{~K} 8+\mathrm{K} 8$ & Tau-Aur & Reipurth et al. (1990) \\
\hline OriNTT5 $69^{2}$ & 4.25 & 0 & $\mathrm{~K} 4$ & Orion Belt & Mathieu (1994) \\
\hline RX J0541.4-0324 ${ }^{2}$ & 4.98 & 0 & $\mathrm{G} 8+\mathrm{K} 3$ & Orion & Covino et al. (2001a) \\
\hline $\mathrm{EKCep}^{2}$ & 4.42 & 0.11 & ${ }^{\dagger} \mathrm{A} 1+\mathrm{G} 5$ & Isolated & Tomkin (1983) \\
\hline $\mathrm{P} 2486^{2}$ & 5.19 & 0.16 & G5 & Trapezium & Mathieu (1994) \\
\hline $\mathrm{W} 134^{2}$ & 6.35 & 0 & ${ }^{\dagger} \mathrm{G} 5+\mathrm{G} 7$ & NGC2264 & Padgett \& Stapelfeldt (1994) \\
\hline OriNTT $429^{2}$ & 7.46 & 0.27 & K3 & Orion Belt & Mathieu (1994) \\
\hline RX J1603.9-3938 & 7.56 & 0 & $\mathrm{~K} 2+\mathrm{K} 3$ & Lupus & This paper; Guenther et al. (2001) \\
\hline $160905-1859^{1}$ & 10.40 & 0.17 & $\mathrm{~K} 1$ & Sco-Cen & Mathieu et al. (1989) \\
\hline VSB126 ${ }^{1}$ & 12.92 & 0.18 & K0 & NGC2264 & Mathieu (1994) \\
\hline $\mathrm{LkCa}^{1}$ & 12.94 & 0.20 & M1 & Tau-Aur & Mathieu (1994) \\
\hline RX J1301.0-7654a ${ }^{2}$ & 13.1 & $0.06(?)^{\dagger \dagger}$ & $\mathrm{K} 3+\mathrm{K} 4$ & Cha & Covino et al. (2001b, in prep.) \\
\hline $\mathrm{AKScO}^{2}$ & 13.60 & 0.47 & $\mathrm{~F} 5+\mathrm{F} 5$ & Sco-Cen & Andersen et al. (1989) \\
\hline RX J1108.8-7519a ${ }^{2}$ & 13.75 & 0.47 & & Cha & Covino et al. (2001b, in prep.) \\
\hline $\mathrm{DQ} \mathrm{Tau}^{2}$ & 15.81 & 0.58 & $\mathrm{~K} 4+\mathrm{K} 4$ & Tau-Aur & Mathieu et al. (1997) \\
\hline $155808-2219^{1}$ & 16.93 & 0.10 & M3 & Sco-Cen & Mathieu (1994) \\
\hline UZ Tau E & 19.1 & 0.28 & & Tau-Aur & Mathieu et al. (1996) \\
\hline $\mathrm{P} 2494^{2}$ & 19.48 & 0.26 & K0 & Trapezium & Reipurth et al. (2000) \\
\hline $\mathrm{P} 1540^{2}$ & 33.73 & 0.12 & $\mathrm{~K} 3+\mathrm{K} 5$ & Trapezium & Marschall \& Mathieu (1988) \\
\hline $162814-2427^{2}$ & 35.95 & 0.48 & $\mathrm{~K} 2$ & $\rho \mathrm{Oph}$ & Mathieu et al. (1989) \\
\hline $\mathrm{P} 1925^{1}$ & 32.94 & 0.55 & K3 & Trapezium & Mathieu (1994) \\
\hline RX J0530.7-0434 ${ }^{2}$ & 40.55 & 0.32 & $\mathrm{~K} 2+\mathrm{K} 2$ & Orion & Covino et al. (2001a) \\
\hline RX J0532.1-0732 ${ }^{2}$ & 46.87 & 0.48 & $\mathrm{~K} 2+\mathrm{K} 3$ & Orion & Covino et al. (2001a) \\
\hline V773 $\mathrm{Tau}^{2}$ & 51.08 & 0.27 & $\mathrm{~K} 2+\mathrm{K} 5$ & Tau-Aur & Welty (1995) \\
\hline $\mathrm{Cru} 3^{2}$ & 58.28 & 0.06 & $\mathrm{~K} 4+\mathrm{K} 5$ & Crux & Alcalá et al. (2001, in prep.) \\
\hline $162819-2423 \mathrm{~S}^{1}$ & 89.1 & 0.41 & G8 & $\rho \mathrm{Oph}$ & Mathieu et al. (1989) \\
\hline $160814-1857^{1}$ & 144.7 & 0.26 & $\mathrm{~K} 2$ & Sco-Cen & Mathieu et al. (1989) \\
\hline $\mathrm{P} 1771^{1}$ & 149.5 & 0.57 & K4 & Trapezium & Mathieu (1994) \\
\hline GWOri $^{1}$ & 241.9 & 0.04 & ${ }^{\dagger} \mathrm{G} 5$ & B30 & Mathieu et al. (1991) \\
\hline HD $98800 \mathrm{~A}^{1}$ & 262.15 & 0.48 & K5 & Isolated & Torres et al. (1995) \\
\hline HD $98800 \mathrm{~B}^{2}$ & 315.15 & 0.78 & $\mathrm{~K} 5+\mathrm{K} 7$ & Isolated & Torres et al. (1995) \\
\hline Haro1-14c $\mathrm{c}^{1}$ & 590.78 & 0.62 & K3 & $\rho \mathrm{Oph}$ & Reipurth et al. (2000) \\
\hline${\text { VSB } 111^{1}}^{1}$ & 879 & 0.8 & G8 & NGC2264 & Mathieu (1994) \\
\hline $045251+3016^{2}$ & 2517.77 & 0.47 & $\mathrm{~K} 5+\mathrm{K} 7$ & Tau-Aur & Steffen et al. (2001) \\
\hline
\end{tabular}

${ }^{1}$ Single-lined spectroscopic binary.

${ }^{2}$ Double-lined spectroscopic binary.

3 Motion of a tertiary component also detected.

† At least one component in the system is out of the mass range considered in Zahn \& Bouchet's (1989) calculations.

$\dagger \dagger$ The preliminary orbital solution does not allow us to assess whether the actual eccentricity is significant or not $(e=$ $0.062 \pm 0.025)$.

Zahn \& Bouchet 1989). Thus, in theory we should expect that a binary system which was circularized by tidal interactions should be also synchronized. In order to check whether or not the rotation rate of both components of RX J1301.0-7654a is already synchronized with the orbital motion we need to estimate the radii for both components.
Covino et al. (2001b, in prep.) derived stellar parameters for both components of RX J1301.0-7654a using the matching technique described in Sect. 2.1. They obtained radii and ages of $R_{\mathrm{a}}=1.8 R_{\odot}$ and $t_{\mathrm{a}}=2 \times 10^{6} \mathrm{yr}$, and $R_{\mathrm{b}}=1.8 R_{\odot}$ and $t_{\mathrm{b}}=2 \times 10^{6} \mathrm{yr}$, for the primary and the secondary, respectively. Taking these values for 


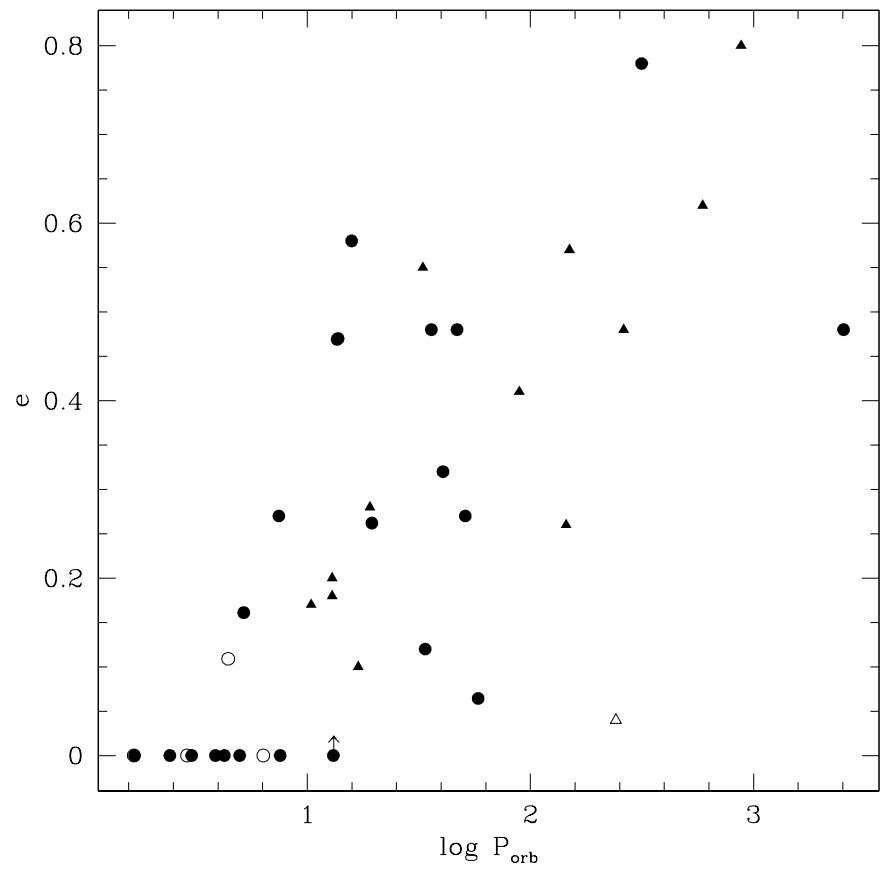

Fig. 2. Orbital eccentricity versus orbital period for the PMS SB with known orbital elements. Circles stand for doublelined spectroscopic binary (SB2) systems and triangles stand for single-lined spectroscopic binary (SB1) systems. Those SB systems having one of the components clearly outside the range of mass explored by Zahn \& Bouchet (1989) are shown as open symbols. The system RX J1301.0-7654a is indicated with an upper arrow since a non-zero eccentricity cannot be ruled out at the moment.

the radii, we can now estimate the synchronous equatorial velocity for each component of RX J1301.0-7654a. Assuming $P_{\text {rot }}=P_{\text {orb }}=13.1$ days, we have $\sim 7 \mathrm{~km} \mathrm{~s}^{-1}$ for the synchronous equatorial velocity for both components. Comparing these values to the projected rotational velocities also given in Covino et al. (2001b) $\left(19 \pm 1 \mathrm{~km} \mathrm{~s}^{-1}\right.$ for both components) we see that the components of RX J1301.0-7654a are rotating at least 2-3 times faster than the synchronous velocity. On this basis, we believe that RX J1301.0-7654a should not be used to define the PMS circularization period, at least until the issue is better understood and the circularity of the orbit confirmed. The system with a circular orbit and the next highest period is RX J1603.9-3938 ( $P_{\text {orb }}=7.56$ days $)$. The synchronous equatorial velocities for this binary computed from the radii given in Table $2\left(\sim 8.4 \mathrm{~km} \mathrm{~s}^{-1}\right.$ and $\sim 8.0 \mathrm{~km} \mathrm{~s}^{-1}$ for the primary and secondary, respectively) are higher than the projected rotational velocities, contrary to the case of RX J1301.0-7654a. Thus, synchronization cannot be ruled out in this case.

Although the hypothesis of synchronous rotation can only be confirmed if the angle $i$ between the line of sight and the orbital plane is known, we can, nevertheless, try to test the plausibility of this hypothesis by using the theoretical masses inferred from the PMS tracks (given in Table 2), and the dynamical minimum masses obtained from the spectroscopic orbit. From the ratio between the
Table 4. Circularization orbital period and age for nonevolved solar mass binaries.

\begin{tabular}{lrlr}
\hline Population & $\begin{array}{c}P_{\text {circ }} \\
\text { (days) }\end{array}$ & $\begin{array}{c}\text { Reference } \\
\text { for } P_{\text {circ }}\end{array}$ & \multicolumn{1}{c}{$\begin{array}{c}\text { Age } \\
(\mathrm{yr})\end{array}$} \\
\hline low-mass PMS & 7.56 & This paper & $1-10 \times 10^{6}$ \\
Pleiades & 7.05 & MRDM92 & $1.2 \times 10^{8}$ \\
Hyades & 8.50 & GG & $6.25 \times 10^{8}$ \\
Praesepe & 8.00 & MM99 & $8 \times 10^{8}$ \\
M 67 & 12.4 & LMMD92 & $5 \times 10^{9}$ \\
Halo & 18.7 & L92 & $1-1.5 \times 10^{10}$ \\
\hline
\end{tabular}

GG - Griffin et al. (1985) and references therein; Stefanik \& Latham (1992).

LMMD92 - Latham et al. (1992).

L92 - Latham et al. (1992a).

MM99 - Mermilliod \& Mayor (1999).

MRDM92 - Mermilliod et al. (1992).

theoretical and dynamical masses, for both components, it turns out that the inclination of the orbit should be around 38 degrees. If we assume that the spin axes are parallel to the axis of the orbit, then the expected projected rotational velocities are about $5 \mathrm{~km} \mathrm{~s}^{-1}$ for both components, remarkably close to the measured $v \sin i$ values (6 and $5 \mathrm{~km} \mathrm{~s}^{-1}$, respectively). Thus, despite the uncertainties involved in deriving the angle $i$ from the comparison between dynamical and theoretical masses, such a test suggests that it is reasonable to assume that both components in RX J1603.9-3938 are rotating synchronously with the orbital motion. Because both synchronization and circularization seem to have been achieved in this system, we will assume here that RX J1603.9-3938 sets the new PMS circularization period at 7.56 days.

\subsection{Are two different mechanisms really needed?}

This new value of the circularization period for the PMS binaries is an important observational constraint supporting Zahn \& Bouchet's (1989) idea that the quasitotality of the orbital circularization occurs during the pre-main sequence phase, primarily near the stellar birthline. According to their results, the circularization period set during the PMS phase for systems with masses ranging from 0.5 to $1.25 M_{\odot}$ is about $7-8.5$ days. In addition, as no further significant circularization occurs during the main-sequence phase, the circularization period for all binary populations (age $<10^{10}$ years) should be quite close to that established during the PMS phase. In Fig. 3, we show the circularization orbital period for the solar-mass binary populations listed in Table 4 . The hatched area corresponds to the circularization orbital period interval predicted by Zahn \& Bouchet (1989) as a result of the PMS tidal circularization. Despite the fact that the circularization periods observed for the PMS, Pleiades, Hyades and Praesepe binary populations agree 


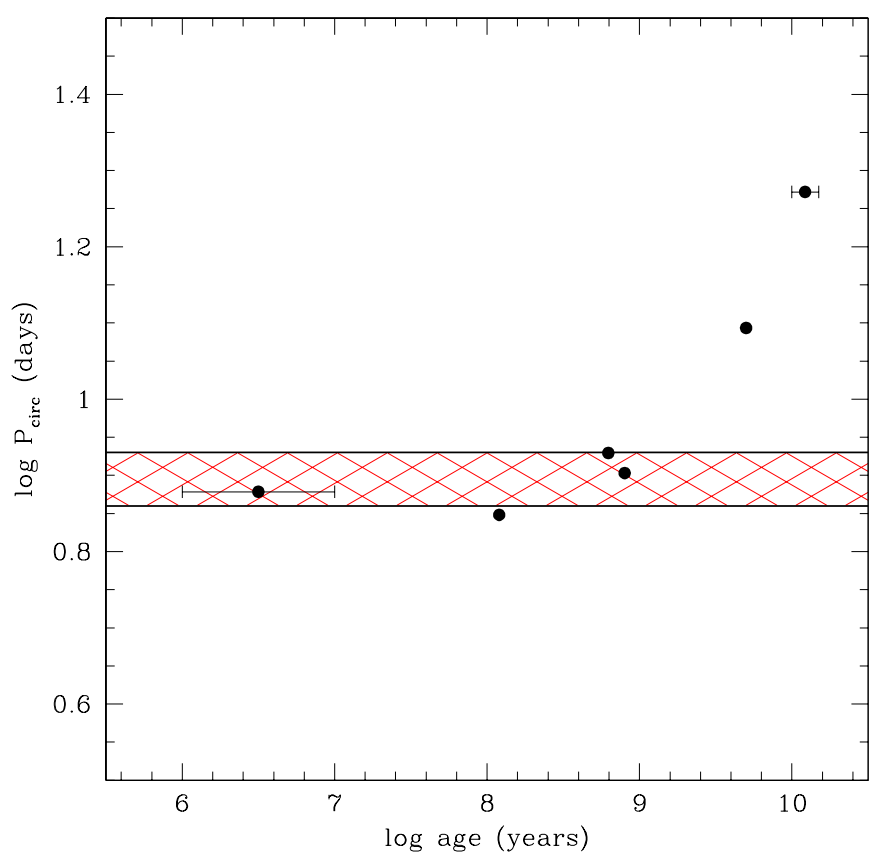

Fig. 3. Circularization orbital period as a function of age for the binary star populations listed in Table 4 . The hatched area corresponds to the circularization orbital period interval predicted by Zahn \& Bouchet (1989) as a result of the PMS tidal circularization. The error bars on the first and last points represent the age spread of the corresponding populations.

with Zahn \& Bouchet's (1989) prediction, the increase of the circularization period for older binary populations (M 67 and Halo stars) is also an observational constraint that cannot be neglected. Mathieu et al. (1992) suggest a hybrid scenario where PMS evolution could indeed establish a circularization period of 7-8.5 days which would be also observed for the binary population in young clusters and, after the lapse of about $10^{9}$ years, main-sequence tidal circularization would become effective. In fact, during the PMS phase the late-type stars have large radii and deep convective envelopes which allow the tidal-torque mechanism to be effective. Once the stars evolve towards the MS these conditions are not present anymore, as a consequence the tidal-torque mechanism looses its strength. Thus, a hybrid scenario requires the existence of a mechanism which will account for the circularization during the MS phase. Such a mechanism has been proposed by Tassoul (see references in Tassoul 1995 and Tassoul 2000), where the angular momentum exchanges are due to hydrodynamical motions (Tassoul 1987). He argues that both mechanisms can not only co-exist but also be complementary, as the hydrodynamical mechanism is quite ineffective during the contraction phase (PMS) and effective on the MS.

The Tassoul hydrodynamical mechanism has been strongly criticized in two papers by Rieutord (1992) and Rieutord \& Zahn (1997) (see also Tassoul's reply in Tassoul \& Tassoul 1997). These authors argue that Tassoul overestimated the strength of the Ekman pumping to spin-up (or spin-down) the fluid in the case where the container is not rigid but the gravitational potential of the star. The correct time-scale for the Ekman pumping to spin-up (or spin-down) the star would be much longer (Eq. (13) and examples in Sect. 4 in Rieutord \& Zahn 1997). The theoretical debate behind the question of whether Tassoul's hydrodynamical mechanism actually operates is beyond the scope of this paper. Instead, let us recall that for the case of M 67 and Halo samples, the time-scales predicted by the tidal-torque mechanism and its variants (i.e., other developments than the original one by Zahn 1977) to circularize orbits up to the observed $P_{\text {circ }}$ are, in both cases, much longer than the age of the sample (Mathieu \& Mazeh 1988; Goldman \& Mazeh 1991, 1992, see also Tassoul 1995, 2000; Goodman \& Oh 1997). This fact does not necessarily lead to the rejection of the tidal-torque mechanism on the MS, it may only mean that future developments, regarding in particular the physical processes that transport angular momentum inside the stars, are needed to reconcile the tidal-torque mechanism with the observations.

Regardless the physics behind the circularization mechanism on the MS, the fact that the present data strongly support the hybrid scenario is an important result per se. As a main (qualitative) consequence of this observational result we can point out that at least up to a given time $t_{\mathrm{MS}}$ (which is of the order of the Hyades age accordingly to the present data), the circularization periods appear to be independent of the age of the samples, as opposed to increasing continuously as a function of time, as previously thought. Thus, they cannot be used either as a clock (Mathieu \& Mazeh 1988) or to test different mechanisms and/or dissipation prescriptions against the data (Mathieu et al. 1992). Nevertheless, as a result of the MS tidal circularization, circularization periods start to increase again as a function of time for coeval samples older than $t_{\mathrm{MS}}$. Consequently, from this time until the moment in which the stellar structure (total mass, internal mass distribution, radii, etc.) can still be considered as constant (i.e., roughly until the end of MS), the circularization period-time relation can be used as a clock and to test different mechanisms and/or dissipation prescriptions for tidal effects.

\subsection{The reliability of the data}

The need for a hybrid scenario lies mainly on two observational facts, the increase of the circularization period for old binary populations and the PMS circularization period. Up to now, the main concern regarding the Zahn \& Bouchet circularization scenario was the lack of observational support. Let us recall that the previous PMS orbital circularization period was set to 6.353 days by the system W134 found by Padgett \& Stapelfeld (1994). However, the dynamical masses implied for this system are about $2 M_{\odot}$, thus suggesting that the mechanism proposed by Zahn \& Bouchet does not have enough time to act due to the shortness of the fully convective phase in such massive stars (Padgett \& Stapelfeld 1994). Thus, the discovery of 
RX J1603.9-3938 is an important observational constraint to the effectiveness of the PMS circularization which gives further support to Zahn and Bouchet's idea.

Due to its relevance, we will discuss the reliability of this PMS circularization period. We argued that, in spite of having circular orbit, the orbital period of the system RX J1301.0-7654a ( $e \approx 0$ and $P_{\text {orb }}=13.1$ days $)$ should not be taken as indicative of the PMS circularization period since neither component is synchronized. However, as the stars contract and the convection zone recedes, tidal braking loses its efficiency, and the conservation of angular momentum in the contracting stars leads to an increase of their rotational velocity which becomes greater than the orbital rate at the moment they arrive on the ZAMS $\left(\sim 10^{7}-10^{8} \mathrm{yrs}\right)$. The ratio between rotational and orbital velocity will depend on the masses of the system (Zahn \& Bouchet 1989). Afterwards, during the MS phase the rotation becomes synchronized again in less than one billion years, and no further significant decrease in the eccentricity occurs (Zahn \& Bouchet 1989). Covino et al. (2001b) estimate the age of this system to be about $\sim 2 \times 10^{6} \mathrm{yr}$. Thus, the system is still far from the ZAMS ${ }^{2}$ and, as a consequence, the argument given above cannot be used to explain its lack of synchronism. At about the age of RX J1301.0-7654a, however, Zahn \& Bouchet (1989) predict that the rotational motion of the components of a binary system composed by two solar mass stars should already start to become unlocked with the orbital motion (see their Fig. 1). Even though, the lack of synchronism by a factor 2-3 observed in RX J1301.0-7654a is higher than that expected according Zahn \& Bouchet scenario for a binary system (about 1.4).

Therefore, we consider the orbital period of the system RX J1603.9-3938, as being the one setting the PMS circularization period. We point out, however, that our decision to dismiss the apparently circular orbit of RX J1301.0$7654 \mathrm{a}$ as being a product of orbital circularization due to its lack of synchronism relies upon the theoretical work of Zahn \& Bouchet (1989) which, in its turn, does not take into consideration disks, magnetic fields or mass accretion of the components which are important phenomena altering the angular momentum of both components of the system. Also, the determination of the circularization periods for the young binary populations listed in Table 4 usually relies on only one binary and the discovery of a new binary with a longer orbital period may change the circularization period of the population as was the case for the PMS SB population. The only effective way to overcome this difficult is to study large samples of binaries.

Another important point has to do with the direct comparison of the circularization period for different populations. In other words, are we comparing binary populations with a similar formation history, and if not, to

\footnotetext{
2 A preliminary analysis of the composite spectra of RX J1301.0-7654a gives a $\log L / L_{\odot} \sim 0.1$ for both components. A detailed analysis will be presented in Covino et al. (2001b).
}

what extent can the observed circularization periods be compared? In the tidal-torque mechanism the time-scale for the circularization will depend on the ratio $(a / R)^{8}$, where $a$ is the semi-major axis and $R$ the stellar radius, on the parameter $\lambda_{\text {conv }}$, which is related to depth of the convective zone (Zahn 1989), and on $q$, the mass ratio. Duchêne et al. (1998) have recently suggested that the binary frequency distribution as a function of the orbital period could depend on the initial conditions. Further supporting this, the results of Brandner \& Köhler (1998) seem to indicate that even the shape of the distribution could depend on the physical properties of the parental cloud. Therefore, it seems that the initial distribution of semimajor axes depends on the environment. This leads to the possibility that some binary populations may be born with larger fractions of very close systems (say, $P_{\text {orb }} \leq 15$ days) compared to other populations. In that case, because of the denser sampling of orbital periods near the critical value, the observed circularization period would tend to be longer than in populations with few binaries in this regime. At the moment, based on the existing data for main sequence binaries in the solar neighborhood (Duquennoy \& Mayor 1991) and for PMS binaries (Mathieu et al. 1992) in the range of separation corresponding to $P_{\text {orb }}<100$ days, the evidence indicates a very similar fraction of spectroscopic binaries of about $10 \%$ in both samples. Thus, it seems unlikely that a given binary population has a longer circularization period due to the fact that more shortperiod binaries were initially formed.

The other parameters $\left(R, \lambda_{\text {conv }}\right)$ are related to the stellar mass. Zahn \& Bouchet (1989) tested the sensitivity of $P_{\text {circ }}$ to the stellar mass and mass ratio. They predict that a little increase of $P_{\text {circ }}$ with mass and with mass ratio should occur $\left(P_{\text {circ }}\right.$ ranging from 7.28 up to 8.54 days). Zahn \& Bouchet also noted that, if the circularization period as a function of the mass is known, we could in principle constrain both the accretion rate and deuterium abundance since the mass-radius relation given by Stahler (1988) depends on these quantities and so does $P_{\text {circ }}$. Nevertheless, at least in a first moment, this spread in $P_{\text {circ }}$ due to the stellar mass can be neglected since not enough data are available to allow to determine $P_{\text {circ }}$ per mass bin.

As a last important point, from Table 3 we note the existence of three systems with orbital periods shorter than the new circularization period of 7.56 and still having an eccentric orbit. According to Zahn \& Bouchet (1989) such systems should not exist since the tidal circularization occurring near the birthline would be strong enough to circularize their orbits. Why are these orbits still eccentric? The answer is not actually known. One possibility to explain the eccentric orbits of these short-period systems might be the pertubations caused by a small mass companion (e.g., Mazeh 1990) or induced by the presence of disks (e.g., Lubow \& Artymowicz 1992). Regarding this later possibility, Artymowicz et al. (1991) studied how a circumbinary disk changes the orbital elements of a central binary system. Their main result is that in general the 
semi-major axis decreases and the eccentricity of the binary is forced to grow. Lubow \& Artymowicz (1992) reinvestigated the problem and concluded that for binaries with small initial eccentricities $(e<0.2)$, the eccentricity will rapidly grow, while for binaries with initial higher eccentricities $(e>0.5)$ the outcome will depend on other parameters of the problem. This means that a PMS binary system possessing a disk of about $1 \%$ of the total mass of the central binary will start to experience the effects of pure tidal circularization (i.e., without competing effects that will tend to increase eccentricity) only after the dissipation of its disk. As a consequence, binaries that preserve their disk for long time-scales (say $1-10 \times 10^{6}$ years) will probably arrive on the ZAMS with high eccentricities as they do not have enough time to circularize their orbits. Among the four confirmed CTTS, three of them, DQ Tau, AK Sco and UZ Tau E, have short-period $P_{\text {orb }}<20$ days and moderate eccentricities while the other, GW Ori has a period of 241.9 days and eccentricity less than 0.1! Clearly, more observational effort must be made in finding more PMS spectroscopic binaries of both types (WTTS and CTTS) which will enable us to have an $e-\log P$ diagram for each type. In addition, surveys devoted to derive disk properties like the mass distribution profile and accretion behavior (e.g., Jensen \& Mathieu 1997; Duchêne et al. 1999; McCabe \& Ghez 2000) will provide important observational constraints to better understand the orbital evolution in a circumbinary and/or circumstellar environment.

\section{Summary and conclusions}

In this paper we have reexamined the issue of the determination of the circularization period for the PMS stars and discussed its relevance to constrain tidal circularization scenarios. In particular, we have presented orbital and stellar parameters for the spectroscopic binary system RX J1603.9-3938. Based on its lithium content, age estimation and barycentric velocity we conclude that both components of this system are bona-fide PMS stars.

Next, we updated the $e-\log P$ diagram for the lowmass PMS stars. Special attention was accorded to the PMS system RX J1603.9-3938 with $P_{\text {orb }}=7.56$ and circular orbit because it sets the circularization period for the PMS binary population. This new circularization period supports Zahn \& Bouchet's (1989) result that tidal circularization during the pre-main sequence will circularize orbits with period up to 7.0-8.5 days. However, the apparently circular orbit of a second system with an orbital period of $P_{\text {orb }}=13.1$ days (RX J1301.0-7654a) poses an ambiguity in the sense that it is not clear whether or not its circular orbit is a result of the PMS tidal circularization. More observations of PMS spectroscopic binaries are needed to further test whether the PMS tidal circularization scenario proposed by Zahn \& Bouchet (1989) needs to be revised to account for other physical phenomena in addition to tidal-torque interactions due to eddy viscosity.
The time dependence of the circularization period for different binary populations indicates a qualitative change at about the age of the Hyades or Praesepe clusters. All younger populations appear to have $P_{\text {circ }}$ around 7 or 8 days, independent of age. This suggests that the orbital evolution in these young populations is a result of tidal circularization during the PMS phase. For older binary populations, on the other hand, the increase in the circularization period with age is evident.

Our main conclusions are that: i) PMS tidal circularization does occur and it seems to be responsible for the circularization period observed in the young clusters up to the age of Praesepe. More data are needed to constrain the efficiency of the tidal interaction during the PMS phase and to better understand the role of other phenomena which may cause angular momentum transfer such as disk interaction, magnetic fields, and accretion; and ii) orbital circularization is still occurring during the MS phase, contrary to Zahn E6 Bouchet's (1989) idea that nearly all tidal circularization occurs during the PMS phase. Both support Mathieu et al.'s (1992) suggestion that a hybrid scenario (PMS plus MS tidal circularization) could explain the observed circularization periods as a function of age. However, which mechanism is supposed to account for the continuous circularization on the MS is still an open question. Tassoul's hydrodynamical mechanism seems to be an alternative (Tassoul 1995 and references therein), although it has been the subject of intense debate on whether or not it can operate (Rieutord 1992; Rieutord \& Zahn 1997; Tassoul \& Tassoul 1997). Further efforts on that front would be important to improve our understanding, in particular, of the physical processes that transport angular momentum inside the stars.

Acknowledgements. CHFM acknowledges grants from CNPQ Proc. 200614/96-7 (NV). EC and JMA acknowledge financial support by the Italian Ministero dell'Università e della Ricerca Scientifica e Tecnologica. This research has made use of the Simbad database, operated at CDS, Strasbourg, France. We thank the anonymous referee for his comments which helped to improve the paper.

\section{References}

Andersen, J. 1975, A\&A, 44, 445

Andersen, J., Lindgren, H., Hazen, M. L., \& Mayor, M. 1989, A\&A, 219, 142

Artymowicz, P., Clarke, C. J., Lubow, S. H., \& Pringle, J. E. 1991, ApJ, 370, L35

Baraffe, I., Chabrier, G., Allard, F., \& Hauschildt, P. H. 1998, A\&A, 337, 403

Brandner, W., \& Köhler, R. 1998, ApJ, 499, L79

Covino, E., Catalano, S., Frasca, A., et al. 2000, A\&A, 361, L49

Covino, E., Melo, C. H. F., Alcalá, J. M., et al. 2001a, A\&A, 375,130 
Casey, B., Mathieu, R. D., Suntzeff, N. B., Lee, C. W., \& Cardelli, J.A . 1993, AJ, 105, 2276

D'Antona, F., \& Mazzitelli, I. 1997, in proceedings of Cool stars in clusters and associations: Magnetic activity and age indicators Me.m. SAIT 68, ed. G. MIcela, R. Pallavicini, \& S. Sciortino, 807

de Jager, C., \& Nieuwenhuijzen, H. 1987, A\&A, 177, 217

de la Reza, R., Quast, G., Torres, C. A. O., et al. 1986, New insights in Astrophysics, ESA SP-263, 107

Duchêne, G., Bouvier, J., Simon, T., Close, L., \& Eislöffel, J. 1998, in CHFT Users Meeting, ed. P. Martin, \& S. Rucinski, 165

Duchêne, G., Monin, J.-L., Bouvier, J., \& Ménard, F. 1999, A\&A, 351, 954

Duquennoy, A., \& Mayor, M. 1991, A\&A, 246, 559

Duquennoy, A., Mayor, M., \& Mermilliod, J.-C. 1992, Binaries as tracers of stellar formation, ed. Duquennoy \& Mayor (Cambridge University Press), 52

Goldman, I., \& Mazeh, T. 1991, A\&A, 376, 260

Goldman, I., \& Mazeh T. 1992, Binaries as tracers of stellar formation, ed. A. Duquennoy, \& M. Mayor (Cambridge University Press), 52

Goodman, J., \& Oh, S. P. 1997, ApJ, 486, 403

Griffin, R. F., Griffin, R. E., Gunn, J. E., \& Zimmerman, B. A. 1985, AJ, 90, 609

Guenther, E. W., Torres, G., Batalha, N., et al. 2001, A\&A, 366,965

Jensen, E., \& Mathieu, R. D. 1997, AJ, 114, 301

Latham, D. W., Mazeh, T., Stefanik, R., et al. 1992a, AJ, 104, 774

Latham, D. W., Mathieu, R. D., Milone, A. E., \& Davis, R. 1992, Evolutionary Processes in Interacting Binary Stars, ed. Y. Kondo, R. F. Sistero, \& R. S. Polidan, IAU Symp., 151,471

Lee, C. W., Martin, E. L., \& Mathieu, R. D. 1994, AJ, 108, 1445

Lubow, S., \& Artymowicz, P. 1992, Binaries as tracers of stellar formation, ed. Duquennoy, \& Mayor (Cambridge University Press), 145

McCabe, C., \& Ghez, A. 2000, Poster Proceedings of IAU Symp. 200, Birth and Evolution of stars, ed. Bo Reipurth, \& Hans Zinnecker, 25

Marschall, L., \& Mathieu, R. D. 1988, AJ, 96, 1956

Mathieu, R. D. 1994, ARA\&A, 32, 465

Mathieu, R. D., Adams, F. C., \& Latham, D. W. 1991, AJ, 101,2184

Mathieu, R. D., \& Mazeh, T. 1988, ApJ, 326, 256
Mathieu, R. D., Martin, E. L., \& Magazzù, A. 1996, AAS Meet., 108, 60.05

Mathieu, R. D., Duquennoy, A., Latham, D. W., et al. 1992, Binaries as tracers of stellar formation, ed. Duquennoy, \& Mayor (Cambridge University Press), 278

Mathieu, R. D., Stassun, K., Basri, G., et al. 1997, AJ, 113, 1841

Mathieu, R. D., Walter, F. M., \& Myers, P. C. 1989, AJ, 98, 987

Mazeh, T. 1990, AJ, 99, 675

Mayor, M., \& Mermilliod, J.-C. 1984, Observational tests of the Stellar Evolution Theory, ed. A. Maeder, \& A. Renzini (Reidel, Dordrecht), 411

Mermilliod, J.-C., \& Mayor, M. 1999, A\&A, 352, 479

Mermilliod, J.-C., Rosvicj, J. M., Duquennoy, A., \& Mayor, M. 1992, A\&A, 265, 513

Padgett, D. L., \& Stapelfeldt, K. R. 1994, AJ, 107, 720

Pasquini, L., Cutispoto, G., Gratton, R., \& Mayor, M. 1991, A\&A, 248, 72

Reipurth, B., Lindgren, H., Nordström, B., \& Mayor, M. 1990, A\&A, 235, 197

Reipurth, B., Lindgren, H., Mayor, M., \& Mermilliod, J.-C. 2000, Birth and Evolution of stars, ed. Bo Reipurth, \& Hans Zinnecker, Poster Proc. IAU Symp., 200, 25

Rieutord, M. 1992, A\&A, 259, 581

Rieutord, M., \& Zahn, J.-P. 1997, ApJ, 474, 760

Simon, M., Dutrey, A., \& Guilloteau, S. 2000, ApJ, 545, 1034

Stahler, S. W. 1988, ApJ, 332, 804

Stefanik, R., \& Latham, D. 1992, IAUC, 135, 173

Steffen, A., Mathieu, R. D., Lattanzi, M. G., et al. 2001, AJ, 122,997

Tassoul, J.-L. 1987, A\&A, 322, 856

Tassoul, J.-L. 1995, ApJ, 444, 338

Tassoul, M., \& Tassoul, J.-L. 1997, ApJ, 481, 363

Tassoul, J.-L. 2000, Stellar Rotation (Cambridge University Press)

Tomkin, J. 1983, ApJ, 271, 717

Torres, G., Stefanik, R. P., \& Latham, D. W. 1995, ApJ, 452, 870

Zahn, J.-P. 1966, Ann. Astrophys., 29, 489

Zahn, J.-P. 1977, A\&A, 57, 383

Zahn, J.-P. 1989, A\&A, 220, 112

Zahn, J.-P., \& Bouchet L. 1989, A\&A, 223, 112

Welty, A. D. 1995, AJ, 110, 776

Wichmann, R., Covino, E., Alcalá, J. M., et al. 1999, MNRAS, 307, 909 